MATEC Web of Conferences 17, 01006 (2014)

DOI: $10.1051 /$ matecconf/ 20141701006

(C) Owned by the authors, published by EDP Sciences, 2014

\title{
Retrospection on the Design of Pedestrian Pathways in a Public Area Interface
}

\author{
S. Naghavi ${ }^{1}$, A.H. Abdul Hamid ${ }^{2, a}$ \\ ${ }^{1,2}$ School of Housing, Building and Planning, Universiti Sains Malaysia, 11800, Penang, Malaysia
}

\begin{abstract}
Some architectural features that cater to human needs, either aesthetically or functionally, include imageability, enclosure, human scale, transparency, and complexity. However, these features cannot be reasonably measured to test the link between design and behaviour. This study looks at the need for maintaining mosque sanctums while allowing the free flow of pedestrians on a university campus. Pedestrian movements are measured through two methods, either the "Thumb Method" or "Pixel Counting," to recognize routes that require improved design. The results of this study showed that pedestrian movements form a basis for design characteristics which also help to clarify route directions for pathway design.
\end{abstract}

\section{Introduction}

According to Great Streets [1]: "Knowing the rhythm of a street is to know who may be on it, or at a certain place along it during a given period; knowing who can be seen there or avoided." Another well said statement that goes along nicely is, "Great cities start with great pedestrian environments." This supports the notion that walking is the most universal form of transport and when streets are designed to prioritize pedestrians, health, economic activity and safety all improve, which would indeed be feasible in a scalable university environment. A pedestrian network should be thoughtfully and artistically designed to draw more people to walk for both utility and pleasure. It should also offer choices of pleasant and interesting routes. Design plays a vital role in the creation of a pedestrian network. At the street level, landscaping which faces human activity and provides aesthetic comfort can influence the way people perceive the neighbourhood as well as their behaviour and movements. Moving up from the street level, porches, overhangs and other architectural features offer protection from heat and rain while reducing energy usage. A study by the Ewing team in 2006 identified five areas that could be reasonably measured to test the link between design and behaviour: imageability, enclosure, human scale, transparency, and complexity [2], [3].

Architecture and its features are able to cater to human needs either aesthetically or functionally. This particular study focuses on the combination of Islamic and Modern Architecture with Technology, which is a blend that has been used countless times all over the world in magnificent, prestigious structures. As an inseparable part of Islamic architecture, gardens encircle every structure at a site. However, the mosque area at Universiti Sains Malaysia suffers from an uneven flow of pedestrian traffic as well as insignificant routes; both of which lead to crowding and unnatural movement on the main walkways when people try to use the pedestrian door by the main gate.

\footnotetext{
${ }^{a}$ Corresponding author : hilcom@usm.my
} 
Consequently, the inner sanctum of the mosque as a holy place is interfered with. This is a situation that should be rectified through a general, purpose-built plan. Accordingly, some related areas that are established in this research are as follows:

\subsection{Regenerated Walkways}

This study assesses people's walking behaviour throughout different periods of the day and different days of week, either weekends or weekdays, to reach a schematic walking path which can lead to the selection of more popular walkways.

\subsection{Clarity of Routes}

One of the proposed goals of this research is to detect the characteristics that relate walkability and walking behaviour to building environment. Places where form, layout, and signage make them easy to understand are likely to both function well and to be pleasant to live in or visit.

\subsection{Mosque Sanctum}

Another significant aim of this research is to simplify the overall look throughout the mosque area as long as it does not blemish the various religious or cultural elements as are present in multinational and diverse countries like Malaysia.

\subsection{Main Entrance Navigability}

Diversification through conceptualization, rearrangement, and maintenance of surrounded walking areas can increase navigability of the premise as a centre point for Universiti Sains Malaysia. Additionally, enhancing and altering the functionality of the entrance as a welcoming area is another aspect of the design proposed in this paper.

\subsection{Environmental Retrospection}

This research concentrates on observations of environmental features around the mosque which include pavements, parking lots, plants, shades, paving or any other architectural features that help to create a sanctum for the mosque.

Figure 2 is a schematic sketch that portrays a more comprehensible and imaginable plan for the target site of this research. The cured, almost-closed circular shape represents the mosque sanctum which is the main focus of this plan. As previously mentioned, the routes which are shown in the sketch need to be recreated and routed in different patterns along the sides. Changes in direction of paths or shortcuts are two other keys to redesigning these routes in order to increase navigability at the entrance. To vitalize walking, designers and urban planners need to go beyond serviceable access. [4] In order to have a functional network that does not hinder the operation and activities at the nodes, there have to be well designed connections between the various hives of activities, spaces, and outlets. In order to create a network that will be efficient and well used by the pedestrians, the future behaviour of pedestrians in such planned environments should be evaluated [5], [6]. The pedestrians' demands will affect mainly their behaviour. This demand will be varying according to a certain distribution of functionalities which may depend on the pedestrians' objectives. The longer the way to the destination, the greater the probability of a route change.

There is a conceptual framework underlying this study that acts as a foundation to prove the correlation between the physical features of environment and walkability (Figure 3). By examining the first part of this framework, which is the link between physical features and urban design qualities, we hope to contribute to a better understanding of the way physical features of the built environment affect walking behaviour. 



Figure 2. A simplified schema of study main aim

Figure 1. Scope of Study - USM - Main Campus - Jalan Masjid

\section{Methodology}

The methods used to find solutions to the questions and stated problems in this research were as follows:

(1) A walkthrough survey

(2) Passive observations by the researcher

(3) A study of the Regional Mobility Initiatives' data

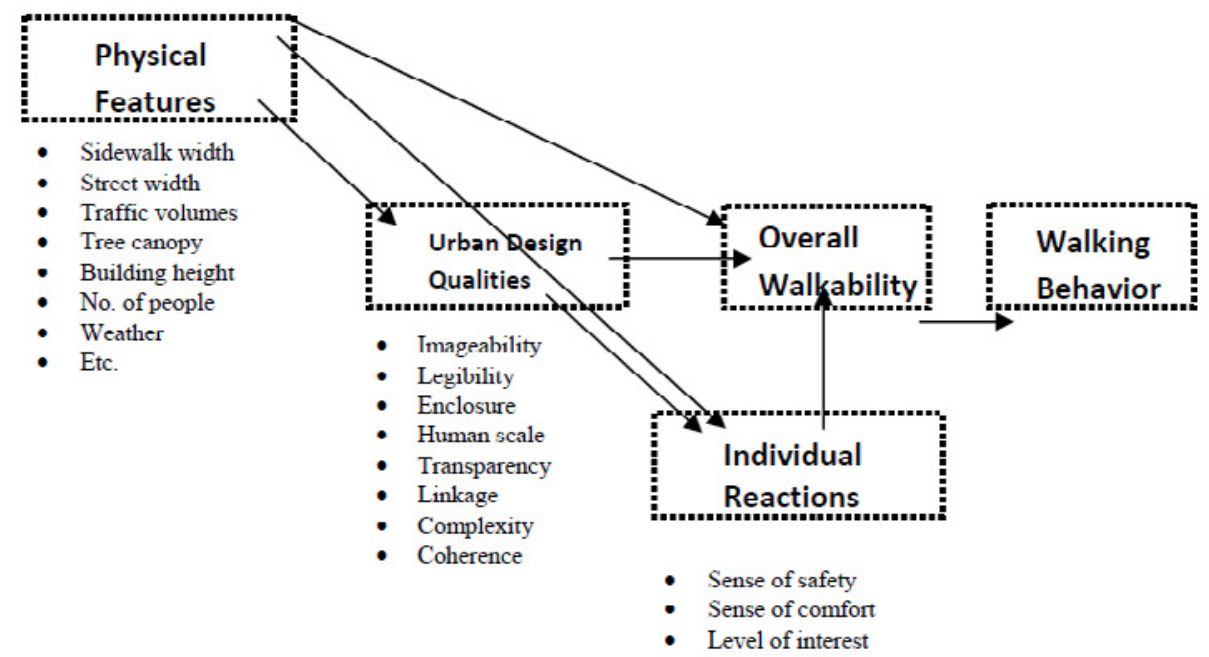

Figure 3. Conceptual Framework 
These methods were used to evaluate the walkability and accessibility of the current walkways as well as to plan the future design. Data collected from these methods was used to analyse the findings and to determine suitable modifications for pavements around the space. Analysis also helped to obtain an in-depth evaluation of the walkability and accessibility of the pedestrian sidewalk along the site area. The author visited places to look for patterns of use on several occasions on different days of the week including holidays, weekends, weekdays, and different times of the day. The most important thing that was looked for at each site was whether or not people were present.

This study required video tape footage for the purpose of observing specific behaviours of pedestrians on sidewalks. The goal was specifically to observe pedestrians' choice of route and how they found their way. Consequences like overall flow, how the flow was affected by pathway design, and how pedestrians chose their routes all affected this urban design plan. These factors also helped pinpoint the most used ways and even other shortcuts that were not necessarily labelled as pavements or pedestrian environments. In every case, videotaped footage was used to observe pedestrians' behaviour. Day and night, with the aid of some helpers, the researcher recorded pedestrians' path choices and routes by following, or 'tracking', individuals and marking the results on a map along with the path they travelled.

Two approaches were considered and evaluated in terms of count accuracy, cost, and ease of deployment. The first approach, named the "Rule of Thumb Method," aimed to estimate the highest number of pedestrians sighted with daily usage in low density pedestrian environments or times. In this study, this method was used for "weekdays and weekends." The numbers were updated based on the amount of movement across selected regions. The second approach to be considered is called "Pixel Counting." In this detection method video frames that were streamed from a low-cost digital camera were carefully processed. Low-level features, such foreground pixels and motion vectors, were used as clues to estimate the number of people passing through the frame. In this research, this method was used on Friday noontime during "Friday Praying" which brings a high-density crowd.

Pixel Counting is a kind of automatic method for counting the people passing by which worked throughout the detection area by using a stereo camera [7], [8]. In the proposed method, stereo images of the passing people were obtained in a series by using stereo cameras located at points 1', 1', 2' and 2" (Figure 1). The frames were divided into sub-regions using video division on different frames that covered up people's traffic. Incoming and outgoing persons could then be measured by counting the pedestrians from each direction (Figure 4).

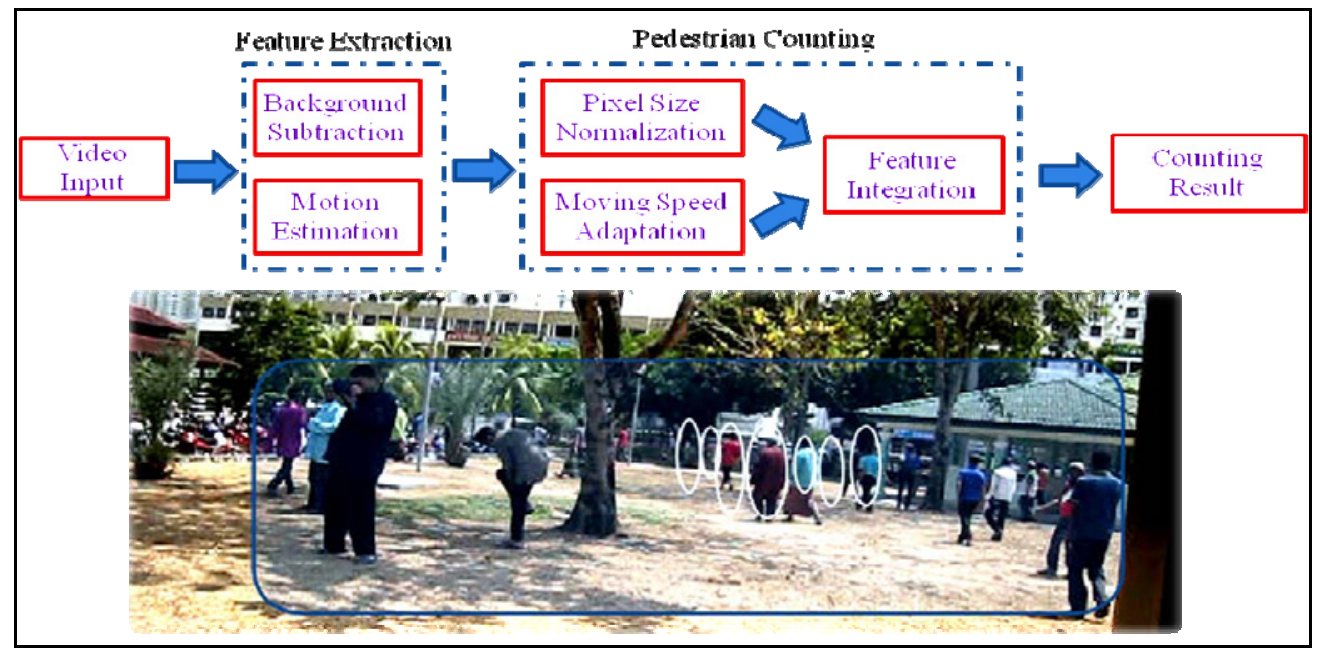

Figure 4. Direction of the pedestrians and extracted features 
As shown in Figure 4, after creating stereo images from the recording, the background was removed from the picture, giving it a transparent look which made the pedestrians stand out in the picture. The counting process then began as mentioned in Figure 4.

\section{Results and Discussion}

Table 1 shows a simplified arrangement of the results of the data analysis. For instance, at the first observation time on Friday noon, at point $1=1^{\prime}+1$ " (Figure 1), 422 pedestrians walked in and 360 people went out of the detection area. This table also shows how many of these people used which route.

Table 1. Analysis of results - Number of pedestrians passing through the detection area

\begin{tabular}{|c|c|c|c|c|c|c|c|c|c|}
\hline \multirow{2}{*}{$\begin{array}{c}\text { No. of } \\
\text { Observation }\end{array}$} & \multirow{2}{*}{ Day } & \multirow{2}{*}{ Site view } & \multirow{2}{*}{ Direction } & \multirow{2}{*}{$\begin{array}{c}\text { No. of } \\
\text { Pedestrian }\end{array}$} & \multicolumn{5}{|c|}{ Routes } \\
\hline & & & & & $\mathrm{A}$ & B & $\mathrm{C}$ & $\mathrm{D}$ & $\mathrm{E}$ \\
\hline \multirow{2}{*}{1} & \multirow{2}{*}{ Friday noon } & \multirow{2}{*}{1} & in & 422 & 147 & 122 & 73 & - & - \\
\hline & & & out & 360 & 140 & 83 & 70 & - & - \\
\hline \multirow{2}{*}{2} & \multirow{2}{*}{$\begin{array}{l}\text { Friday } \\
\text { erening }\end{array}$} & \multirow{2}{*}{1} & in & 42 & 14 & 11 & 6 & - & - \\
\hline & & & out & 98 & 39 & 30 & 17 & - & - \\
\hline \multirow{2}{*}{3} & \multirow{2}{*}{ Weekday } & \multirow{2}{*}{1} & in & 66 & 5 & 11 & 39 & & \\
\hline & & & out & 205 & 16 & 32 & 86 & & \\
\hline \multirow{2}{*}{4} & \multirow{2}{*}{ Weekday } & \multirow{2}{*}{2} & in & 52 & - & . & - & 40 & 9 \\
\hline & & & out & 95 & - & - & - & 66 & 14 \\
\hline \multirow{2}{*}{5} & \multirow{2}{*}{ weekend } & \multirow{2}{*}{1} & in & 59 & 4 & 17 & 35 & & \\
\hline & & & out & 170 & 14 & 21 & 92 & & \\
\hline \multirow{2}{*}{6} & \multirow{2}{*}{ weekend } & \multirow[b]{2}{*}{2} & in & 61 & - & - & - & 45 & 11 \\
\hline & & & out & 83 & - & . & - & 46 & 25 \\
\hline
\end{tabular}

Route detection was the result of preliminary surveillance which picked out 5 routes among all the walkways as those most used by pedestrians (Figure 5). From all the surveys, information obtained suggests that square-away pathways need to be designed in order to encourage people towards the right direction. Moreover, facilitating, diversifying, and distinguishing pathways with the use of diversified structures and operations will help bring the desired and needed changes.

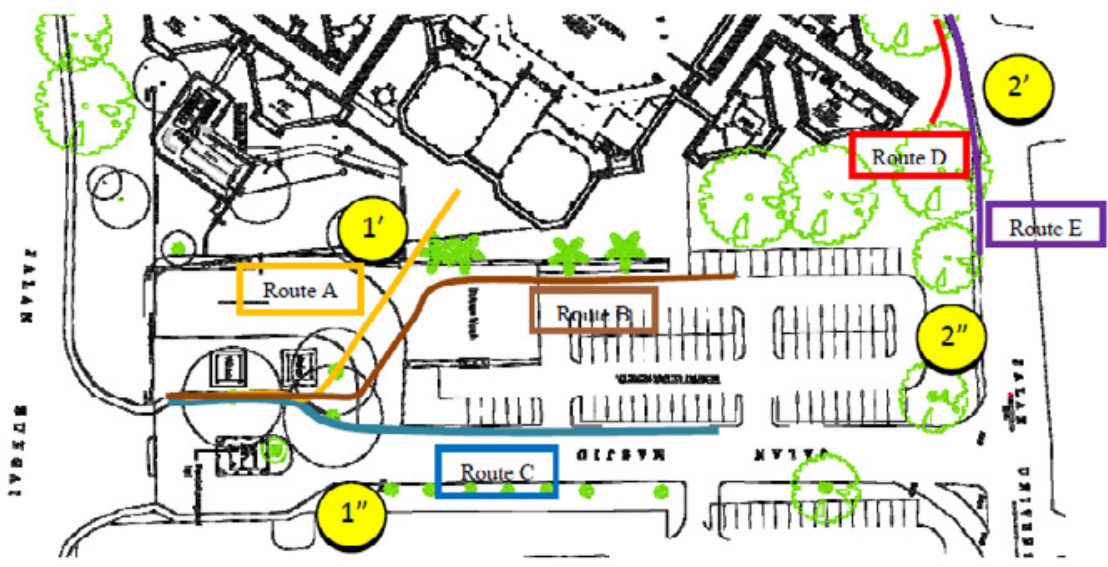

Figure 5. Analysis of results - Most used routes detected on the proposed site 


\section{Conclusion}

As a result of this research, a simplified and new perspective towards measuring pedestrian movements has been gained. It was possible to develop an overall understanding of the pattern of pedestrian's movements and an estimation of their quantity with the help of some simple tools like a basic video camera to videotape movements in crowded places or in peak hours of the day and a camera for photographing areas with fewer pedestrians passing by or during times or days when there were not many people in the target area. Further modification using software like Adobe Photoshop or Illustrator also came in useful during the data collection process. Undoubtedly, in a place armed with more sufficient facilities like CCTV and the possibility to devote more observation time, the results would be even more substantial. The ability to measure and evaluate pedestrian movement demands will help designers in forming the most appropriate pathways to maximize the flow of pedestrians yet keep the mosque sanctum area free from disruption. To legitimize all of this information, proof of the functionality of the devised plan should be prepared and submitted. Hence, further design details for the proposed walkway plan are forthcoming.

\section{References}

1. A. B. Jacobs, "Great Streets," vol. 1, no. 3, Sep. 1993.

2. R. Ewing, S. Handy, R. C. Brownson, O. Clemente, and E. Winston, "Identifying and Measuring Urban Design Qualities Related to Walkability," pp. 223-240, 2006.

3. R. Ewing and S. Handy, "Measuring the Unmeasurable: Urban Design Qualities Related to Walkability," J. Urban Des., vol. 14, no. 1, pp. 65-84, Feb. 2009.

4. A. Forsyth and M. Southworth, "Cities Afoot—Pedestrians, Walkability and Urban Design," J. Urban Des., vol. 13, no. 1, pp. 1-3, Feb. 2008.

5. B. McLaughlin, "Creating a pedestrian behaviour prediction model from an empirical study of the Xu Jia Hui pedestrian network in Shanghai."

6. K. Helbing, Dirk and Molnar, Peter and Farkas, Illes J and Bolay, "Self-organizing pedestrian movement," Environ. Plan. B, vol. 28, pp. 361-384, 2001.

7. K. Terada, D. Yoshida, S. Oe, and J. Yamaguchi, "A method of counting the passing people by using the stereo images," Proc. 1999 Int. Conf. Image Process. (Cat. 99CH36348), vol. 2, pp. 338-342, 1999.

8. D. Kong and D. Gray, “A Viewpoint Invariant Approach for Crowd Counting,” 18th Int. Conf. Pattern Recognit., vol. 1, pp. 1187-1190, 2006. 